\title{
Corrigendum
}

\section{Corrigendum to "Expression of TRPC6 in benign and malignant human prostate tissues" by Dan Yue et al.}

\author{
Dan Yue ${ }^{1}$, Yong Wang ${ }^{2}$, Jian-Ying Xiao ${ }^{3}$, Ping Wang ${ }^{2}$, Chang-Shan Ren ${ }^{1}$
}

${ }^{1}$ Cancer Research Institute, First Affiliated Hospital, China Medical University, Shenyang 110001, China

${ }^{2}$ Departments of Urology, Fourth Affiliated Hospital, China Medical University, Shenyang 110032, China

${ }^{3}$ Department of Biochemistry, Liaoning Medical University, Jinzhou 121001, China

Asian Journal of Andrology (2010) 12: 124. doi: 10.1038/aja.2009.82

Correspondence to: Dr Chang-Shan Ren, Cancer Research Institute, First Affiliated Hospital, China Medical University, Shenyang 110001, China.

Fax:+86-24-2326-8468Ｅ-mail: csrencmu@yahoo.com.cn

Received: 26 October 2009 Accepted: 5 November 2009

In published paper by Dan Yue et al. [1], the Table 4 in page 545 was inadvertently misrepresented. The correct number and percentage of TRPC6 positive in BPH should be 9 (45.0). Now, the correct representation of Table 4 should be:

Table 4. Relationship between androgen responsiveness of tumor and TRPC6 protein.

\begin{tabular}{crcr}
\hline Androgen responsiveness & $n$ & Evaluable specimens & TRPC6 positive (\%) \\
\hline BPH & 20 & 20 & $9(45.0)$ \\
AD & 102 & 99 & $82(82.8)$ \\
AI & 34 & 33 & $29(87.9)$ \\
\hline
\end{tabular}

Abbreviations: AD, androgen-dependent; AI, androgen-independent; BPH, benign prostatic hyperplasia; TRPC6, transient receptor potential canonical 6 .

The authors would offer the profoundest apologies to the readers for any confusion and misunderstanding due to our careless and errors.

\section{Reference}

1 Yue D, Wang Y, Xiao JY, Wang P, Ren CS. Expression of TRPC6 in benign and malignant human prostate tissues. Asian J Androl 2009; 11: $541-7$. 